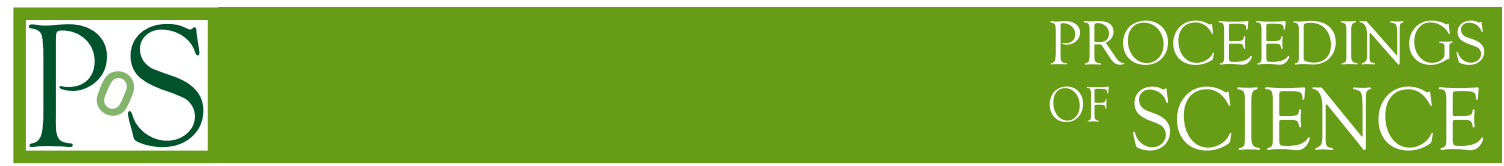

\title{
Physics Program of the Super Tau-Charm Factory
}

\author{
Xiao-Rui Lyu (on behalf of the STCF working group) ${ }^{a, *}$ \\ ${ }^{a}$ University of Chinese Academy Sciences, \\ Beijing 100049, People's Republic of China \\ E-mail: xiaorui@ucas.ac.cn
}

In this talk, I present the potential physics program of the super $\tau$-charm factory (STCF). The STCF is an electron-positron collider with a center-of-mass energy ranging from 2 to $7 \mathrm{GeV}$ and an instantaneous luminosity greater than $0.5 \times 10^{35} \mathrm{~cm}^{-2} \mathrm{~s}^{-1}$. This energy region corresponds to the transition between non-perturbative quantum chromodynamics (QCD) and perturbative QCD. Hence, a large variety of topics in elementary particle physics can be pursued at STCF, including exploring QCD and hadron spectroscopy, precise measurement of electroweak interactions and flavor physics studies as well as searches for the new physics beyond the standard model.

BEAUTY2020

21-24 September 2020

Kashiwa, Japan (online)

\footnotetext{
${ }^{*}$ Speaker
} 


\section{Introduction}

Currently, the only facility operating in the $\tau$-charm energy region is the Beijing Spectrometer (BESIII) at the Beijing Electron Positron Collider (BEPCII) [1], which has significantly advanced the progress of elementary particle physics. A comprehensive description of the physics program and potential of BESIII can be found in Refs. [2, 3]. BESIII has been operating for more than 11 years reliably at its designed luminosity of $10^{33} \mathrm{~cm}^{-2} \mathrm{~s}^{-1}$ at $\sqrt{\mathrm{s}}=3.773 \mathrm{GeV}$ and collected data from 2.0 to $4.95 \mathrm{GeV}$, with an integrated luminosity of more than $30 \mathrm{fb}^{-1}$. The BEPCII/BESIII facility will complete its mission within 5-10 years. So a next-generation facility that continues and extends the research topics in the relevant energy region with significantly enhanced sensitivity is definitely necessary. A Super $\tau$-Charm facility (STCF), with a luminosity that is two orders of magnitude higher, would be a natural extension and a viable option. The successful construction and operation of an STCF will play a crucial role in leading the high-intensity frontier of elementary particle physics worldwide.

The proposed STCF [4] is an electron-positron collider to be situated in China, with separated electron and positron rings and symmetric beam energy, as shown in Fig. 1(a). It is designed to have an energy spanning between 2 and $7 \mathrm{GeV}$, with a peak luminosity of at least $0.5 \times 10^{35} \mathrm{~cm}^{-2} \mathrm{~s}^{-1}$, optimized at a center-of-mass energy of $4 \mathrm{GeV}$. The proposed design would leave space for higher luminosity upgrades and for the implementation of a polarized $e^{-}$beam in a future second phase [5]. The STCF detector [6] will have a near $4 \pi$ solid-angle acceptance, shown in Fig. 1(b), and features low noise, high detection efficiency and resolution as well as excellent particle-identification capabilities.

The STCF conceptual design report is expected to be released in 2021 and the technical design report will be ready by 2024 . The construction of STCF facility is scheduled during the years of 2024-2030. Afterwards, STCF will operate for 10 years during the period 2031-2040. The second phase with an upgrade of electron beam polarization will be realized during 2041-2042 and will continue for 5 years of data taking. The budget of STCF pre-research is estimated to be about 250 million Chinese Yuan and that of construction to be about 4.5 billion Chinese Yuan. There are a few options for the experimental site under consideration and the final decision will be made during the coming years.

The planned STCF would be a copious source of $\tau$-leptons, charmed mesons and baryons, and strange hyperons and therefore address a very broad range of physics topics, as indicated in Fig. 2, such as tests of QCD, (exotic) hadron spectroscopy, flavor physics, precision tests of the electroweak sector of the standard model (SM) and searches for new physics beyond the SM. Without question, the energy region of $5 \sim 7 \mathrm{GeV}$ has a great physics potential, in particular for the studies of singly or doubly charmed baryons, and pentaquark states $P_{c}(4450)^{+}$, but where there currently exist very few experimental results. The expected integrated luminosity for data taking at STCF per year is expected to be more than $1 \mathrm{ab}^{-1}$. A possible data-taking plan for the STCF, along with the expected size of the produced samples, is shown in Table 1. In the following sections, some key aspects of the physics program are highlighted. 


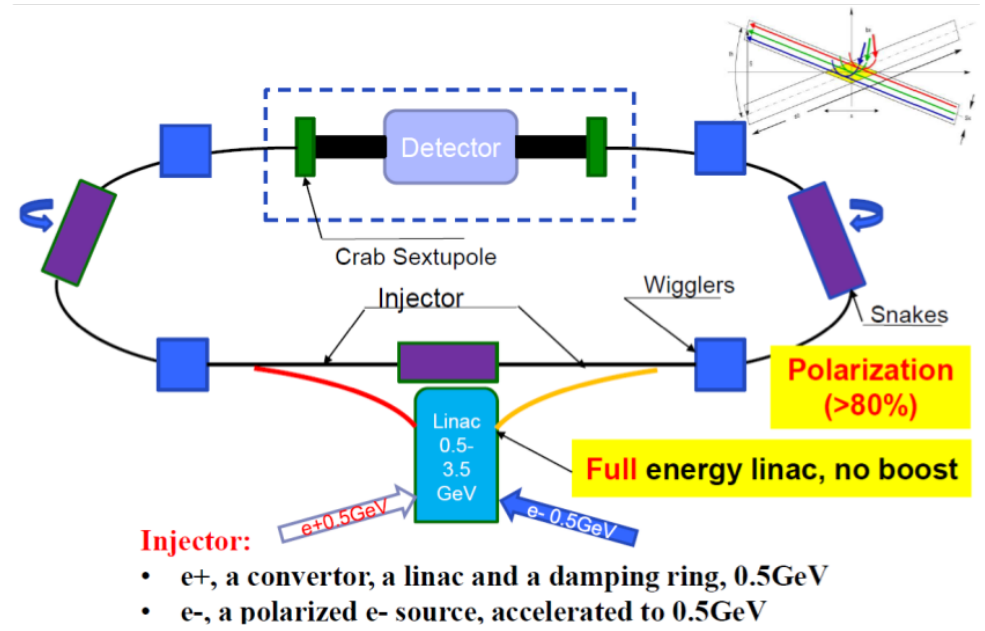

(a)

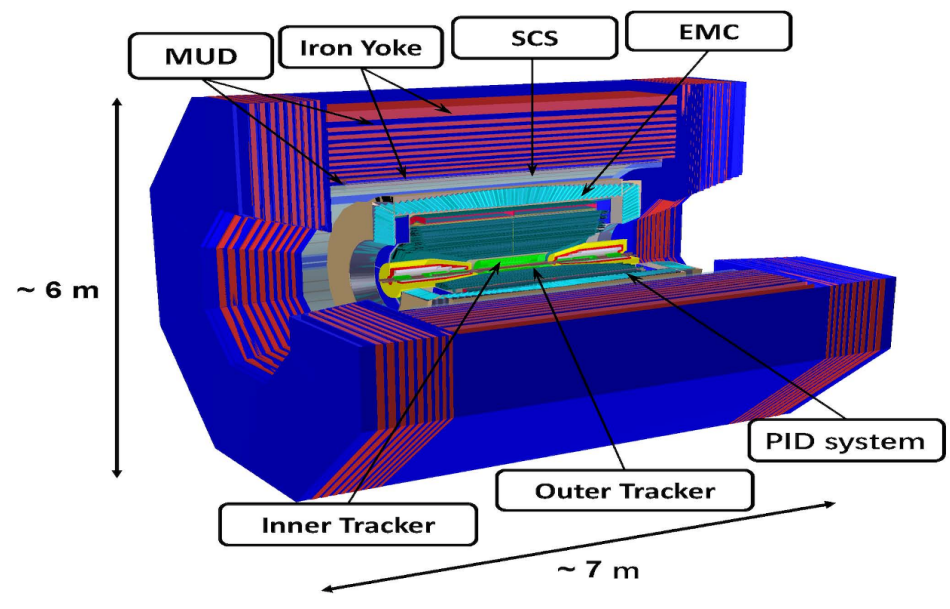

(b)

Figure 1: A schematic diagram of the STCF collider (a) and the detector design (b).

\section{Charmonium(-like) spectroscopy}

BESIII has played a leading role in the discovery and study of so-called $X Y Z$ hidden-charm states, which can not be accommodated in standard charmonium spectroscopy, as shown in Fig. 3. BESIII has accumulated data at the $\psi(3686)$ peak and at energy values between 4.0 and $4.95 \mathrm{GeV}$ to study charmonium spectroscopy and the charmonium-like $X Y Z$ mesons. Analyses of this sample have yielded some of BESIII's most remarkable results such as the discoveries of the charged charmonium-like states, $Z_{c}(3900)[7,8]$ and $Z_{c}(4020)$ [9, 10], the $Z_{c s}(3985)$, the first example of a charmonium-like state with non-zero strangeness [11], an anomalous line shape for the $Y(4260)$ resonance [12] and a large partial decay width for the radiative process $Y(4260) \rightarrow \gamma X(3872)$ [13].

As can be seen from Table 1, at STCF not only large data samples of conventional $J / \psi$ and $\psi(3686)$ particles can be collected, but also copious $X Y Z$ event samples will be produced. These large data samples will enable detailed studies of the $X Y Z$ meson properties, searches for new 


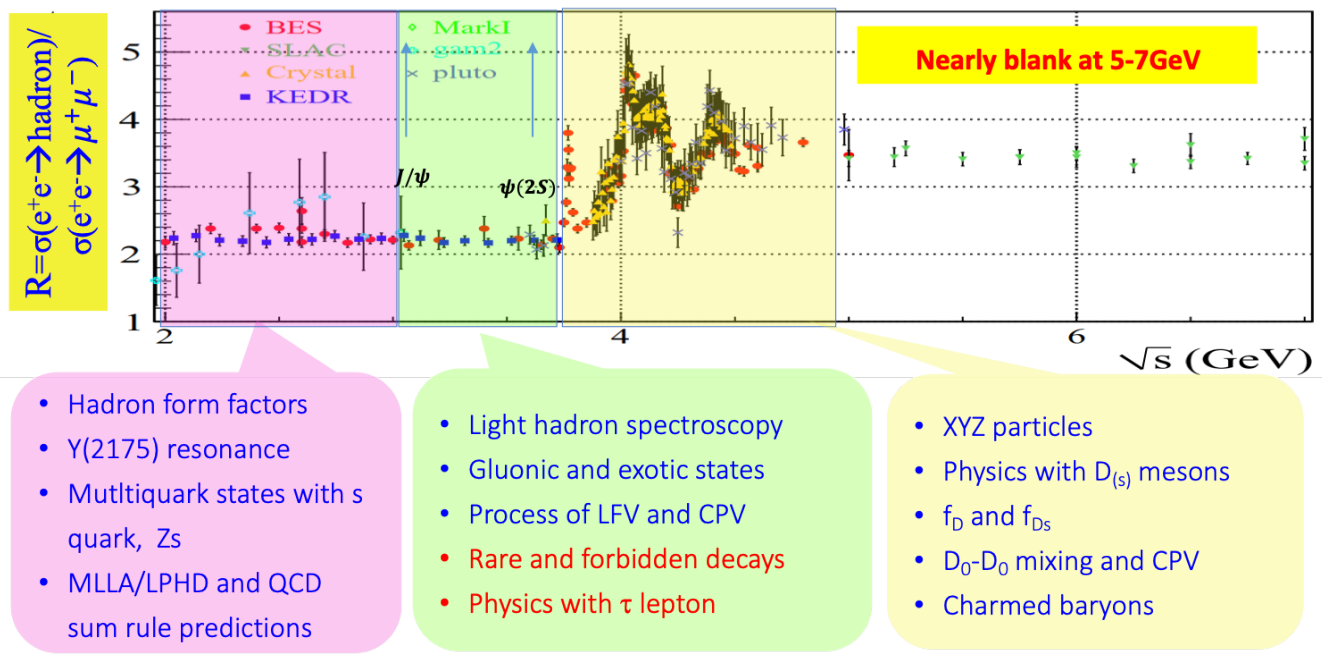

Figure 2: $R$ value distribution and the potential physics topics in the energy region between 2 and $7 \mathrm{GeV}$.

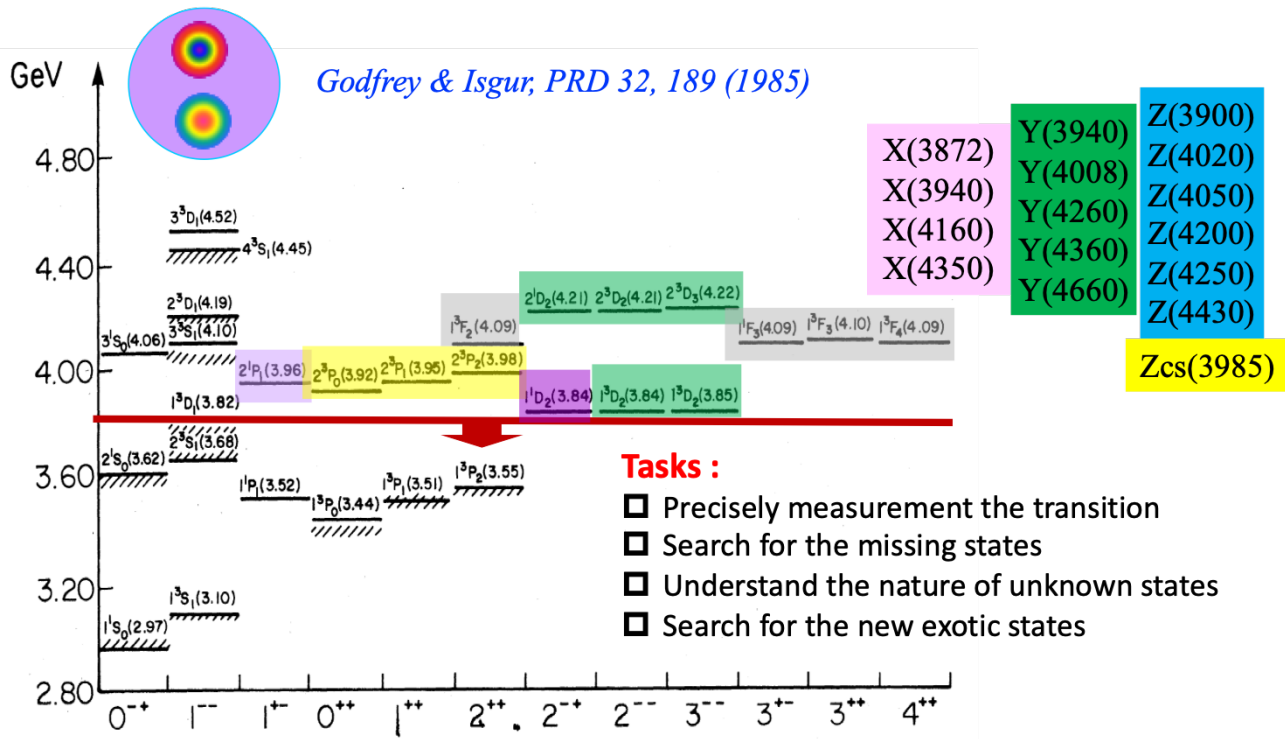

Figure 3: Charmonium spectroscopy and the observed non-conventional charmonium-like states.

exotic states and charmonium rare decays, precise measurements of the mass, width and transitions of different charmonium(-like) states.

\section{Charm decays}

A high-luminosity STCF, which is capable of producing about $10^{9} \sim 10^{10}$ quantum-coherent $D^{0} \bar{D}^{0}$ meson pairs and $D_{(s)}^{+}$mesons, more than $10^{8} \Lambda_{c}^{+}$baryons as well as heavier charmed baryons, will be an important low-background facility to test the SM and probe for new physics. In particular, it will serve as an excellent laboratory to determine the Cabibbo-Kobayashi-Maskawa (CKM) matrix 
Table 1: The expected sizes of events per year at different energy points with integrated luminosity $\mathcal{L}$ at STCF.

\begin{tabular}{|c|c|c|c|c|}
\hline Energy $(\mathrm{GeV})$ & $\mathcal{L}\left(\mathrm{ab}^{-1}\right)$ & Samples & $\sigma(\mathrm{nb})$ & No. of Events \\
\hline 3.097 & 1 & $J / \psi$ & 3400 & $3.4 \times 10^{12}$ \\
\hline 3.670 & 1 & $\tau^{+} \tau^{-}$ & 2.4 & $2.4 \times 10^{9}$ \\
\hline 3.686 & 1 & $\begin{array}{c}\psi(3686) \\
\tau^{+} \tau^{-}\end{array}$ & $\begin{array}{l}640 \\
2.5\end{array}$ & $\begin{array}{l}6.4 \times 10^{11} \\
2.5 \times 10^{9}\end{array}$ \\
\hline 3.770 & 1 & $\begin{array}{c}D^{0} \bar{D}^{0} \\
D^{+} \bar{D}^{-} \\
\tau^{+} \tau^{-}\end{array}$ & $\begin{array}{l}3.6 \\
2.8 \\
2.9\end{array}$ & $\begin{array}{l}3.6 \times 10^{9} \\
2.8 \times 10^{9} \\
2.9 \times 10^{9}\end{array}$ \\
\hline 4.009 & 1 & $\begin{array}{c}D^{* 0} \bar{D}^{0}+c . c . \\
D^{*+} D^{-}+c . c . \\
D_{s}^{+} D_{s}^{-} \\
\tau^{+} \tau^{-}\end{array}$ & $\begin{array}{l}4.0 \\
4.0 \\
0.2 \\
3.5\end{array}$ & $\begin{array}{l}4.0 \times 10^{9} \\
4.0 \times 10^{9} \\
2.0 \times 10^{8} \\
3.5 \times 10^{9}\end{array}$ \\
\hline 4.180 & 1 & $\begin{array}{c}D_{s}^{+*} D_{s}^{-}+\mathrm{c} . \mathrm{c} . \\
D^{* 0} \bar{D}^{* 0} \\
D^{*+} D^{*-} \\
\tau^{+} \tau^{-}\end{array}$ & $\begin{array}{l}0.9 \\
2.5 \\
2.5 \\
3.6\end{array}$ & $\begin{array}{l}9.0 \times 10^{8} \\
2.5 \times 10^{9} \\
2.5 \times 10^{9} \\
3.6 \times 10^{9}\end{array}$ \\
\hline 4.230 & 1 & $\begin{array}{c}J / \psi \pi^{+} \pi^{-} \\
\tau^{+} \tau^{-}\end{array}$ & $\begin{array}{c}0.085 \\
3.6\end{array}$ & $\begin{array}{l}8.5 \times 10^{7} \\
3.6 \times 10^{9}\end{array}$ \\
\hline 4.360 & 1 & $\begin{array}{c}\psi(3686) \pi^{+} \pi^{-} \\
\tau^{+} \tau^{-}\end{array}$ & $\begin{array}{c}0.058 \\
3.5\end{array}$ & $\begin{array}{l}5.8 \times 10^{7} \\
3.5 \times 10^{9}\end{array}$ \\
\hline 4.420 & 1 & $\begin{array}{c}\psi(3686) \pi^{+} \pi^{-} \\
\tau^{+} \tau^{-}\end{array}$ & $\begin{array}{c}0.040 \\
3.5\end{array}$ & $\begin{array}{l}4.0 \times 10^{7} \\
3.5 \times 10^{9}\end{array}$ \\
\hline 4.630 & 1 & $\begin{array}{c}\psi(3686) \pi^{+} \pi^{-} \\
\Lambda_{c} \bar{\Lambda}_{c} \\
\tau^{+} \tau^{-}\end{array}$ & $\begin{array}{c}0.033 \\
0.56 \\
3.4\end{array}$ & $\begin{array}{l}3.3 \times 10^{7} \\
5.6 \times 10^{8} \\
3.4 \times 10^{9}\end{array}$ \\
\hline $4.0-7.0$ & $5-10$ & \multicolumn{3}{|c|}{ fine scan data } \\
\hline
\end{tabular}

elements $V_{c d}$ and $V_{c s}$, to measure $D^{0}-\bar{D}^{0}$ mixing parameters, to probe for CP violation in the charm sector, to search for rare and forbidden charmed hadron decays, and to study other fundamental problems associated with charmed hadrons.

The most precise way to determine $V_{c d}$ and $V_{c s}$ at STCF is via pure-leptonic decays $D_{(s)}^{+} \rightarrow$ $\ell^{+} v_{\ell}$ (for $\left.\ell=e, \mu, \tau\right)$ [14]. The product of the decay constant $f_{D_{(s)}^{+}}$, and $V_{c d(s)}$ is directly accessed by measuring the widths of $D_{(s)}^{+} \rightarrow \ell^{+} v_{\ell}$ decays. Then with the input of $f_{D_{(s)}^{+}}$from Lattice QCD, the value of $V_{c d(s)}$ or $f_{D_{(s)}^{+}}$can be obtained. The projected statistical relative uncertainties of $V_{c d}$ and $V_{c s}$ will be $0.15 \%$ and $0.08 \%$ at STCF, which will improve the current world precision by factors of 20 and 10, respectively. Lepton flavor universality (LFU) can be tested by examining the ratio of the decay rate of $D_{(s)}^{+} \rightarrow \tau^{+} v$ and $D_{(s)}^{+} \rightarrow \mu^{+} v$. At STCF, the statistical precision on these ratios will be comparable to the uncertainties of the predictions in the SM. Hence, it will 
provide meaningful tests on LFU via these channels. Additional LFU test can be performed via semi-leptonic decay modes. Measurements of the ratios of the partial widths of $D^{0(+)} \rightarrow h \mu^{+} v_{\mu}$ over those of $D^{0(+)} \rightarrow h e^{+} v_{e}$ in different $q^{2}$ intervals constitute a complementary test of LFU to those using tauonic decays.

STCF will be a unique place for the study of $D^{0}-\bar{D}^{0}$ mixing and CP violation by means of quantum coherence of $D^{0}$ and $\bar{D}^{0}$ mesons produced at energy points near threshold. A $D^{0} \bar{D}^{0}$ pair can be coherently produced through the reactions $e^{+} e^{-} \rightarrow\left(D^{0} \bar{D}^{0}\right)_{\mathrm{CP}=-}$ at $3.773 \mathrm{GeV}$ and $e^{+} e^{-} \rightarrow D^{0} \bar{D}^{* 0} \rightarrow \pi^{0}\left(D^{0} \bar{D}^{0}\right)_{\mathrm{CP}=-}$ or $\gamma\left(D^{0} \bar{D}^{0}\right)_{\mathrm{CP}=+}$ at $4.009 \mathrm{GeV}$. One may therefore obtain useful constraints on $D^{0}-\bar{D}^{0}$ mixing and CP-violating parameters in the respective decays of correlated $D^{0}$ and $\bar{D}^{0}$ events. For example, the $D^{0}-\bar{D}^{0}$ mixing rate $R_{M}=\left(x^{2}+y^{2}\right) / 2$ can be accessed via the same charged final states $\left(K^{ \pm} \pi^{\mp}\right)\left(K^{ \pm} \pi^{\mp}\right)$ or $\left(K^{ \pm} \ell^{\mp} v\right)\left(K^{ \pm} \ell^{\mp} v\right)$ with a sensitivity of $10^{-5}$ with $1 \mathrm{ab}^{-1}$ data at $3.773 \mathrm{GeV}$. In the $e^{+} e^{-} \rightarrow \gamma D^{0} \bar{D}^{0}$ sample, the charm mixing contribution is doubled compared with the time-dependent (un-correlated) case. With $1 \mathrm{ab}^{-1}$ data at $4.009 \mathrm{GeV}$, it is expected that the measurement sensitivities of the mixing parameters $(x, y)$ will reach a level of $0.05 \%$, and those of $|q / p|$ and $\arg (q / p)$ will be $1.7 \%$ and $1.3^{\circ}$, respectively. These sensitivities are complementary to the future precision measurements foreseen at Belle II and the LHCb upgrades.

The quantum correlation of the $D^{0} \bar{D}^{0}$ system provides a unique opportunity to determine the strong-phase difference between Cabibbo-favored and doubly Cabibbo-suppressed amplitudes. Measurements of the strong-phase difference are well motivated in several aspects: understanding the non-perturbative QCD effects in the charm sector; serving as essential inputs to extract the angle $\gamma$ of the CKM unitarity triangle (UT) at LHCb and Belle II, and relating the measured mixing parameters in hadronic decay $\left(x^{\prime}, y^{\prime}\right)$ to the mass and width difference parameters $(x, y)$ [15]. The most precise method to measure $\gamma$ is based upon the interference between $B^{+} \rightarrow \bar{D}^{0} K^{+}$and $B^{+} \rightarrow D^{0} K^{+}$decays. In the future, the statistical uncertainties of these measurements will be greatly reduced by using the large $B$ meson samples recorded by LHCb and Belle II. Hence, to match the future statistical uncertainty of less than $0.4^{\circ}$ foreseen in the future LHCb upgrade II, STCF would be able to provide important constraints to reduce the systematic uncertainty from $D$ strong-phase to be less than $0.1^{\circ}$ and allow detailed comparisons of the $\gamma$ results from different decay modes.

Furthermore, studies of charmed baryon decays can be extensively carried out using the threshold production data. The absolute measurements of the semi-leptonic and nonleptonic decays of the $\Lambda_{c}^{+}, \Xi_{c}^{+, 0}$ and $\Omega_{c}^{0}$ baryons will be significantly improved at STCF. Priority will be ascribed to the decay asymmetries $\alpha$ in various charm baryon decays, which can be further used to search for CP-violating effects, and the absolute branching fractions of $\Omega_{c}^{0}$ decays. Moreover, measurements of and searches for rare- and forbidden decays of charmed hadrons with up to two orders-of-magnitude improvements in sensitivity could be realized as as part of a search for new physics.

\section{4. $\tau$ physics}

The $\tau$, as the heaviest charged lepton, has many more decay channels than muon, and provides access to new physics beyond the SM. As listed in Table 1, STCF can produce up to $10^{10} \tau^{+} \tau^{-}$pairs at different energy points. Near threshold, one can use data from slightly lower energies to better understand the background, and achieve relatively high efficiency, which make STCF competitive 
with respect to $\tau$ physics studies at Belle II. STCF will tremendously increase the significance in $\tau$-physics measurements and will reach a level of precision which has never been reached before.

Another unique advantage of $\tau$-pairs that are produced near-threshold, is that they are primarily produced in an $s$-wave and, thus, if the electron beam is polarized, this polarization translates nearly $100 \%$ into a well understood polarization of the two final-state $\tau$ leptons [16]. Thus, STCF operation with a polarized electron beam just above the $\tau$-pair threshold would enable a high-sensitivity search for CP-violating asymmetries in $\tau^{-} \rightarrow \pi^{-} \pi^{0} v$ decays [16]. With the $\tau$ threshold data, STCF is also suitable for a high-sensitivity study of the anomalous $(\sim 3 \sigma)$ sign of CP violation in $\tau^{-} \rightarrow K_{S} \pi^{-} v$ decays that was reported by BaBar [17]. A preliminary MC study shows that an uncertainty of $0.06 \%$ on the CP asymmetry in $\tau^{-} \rightarrow K_{S} \pi^{-} v$ can be obtained based on $1 \mathrm{ab}^{-1}$ data at $4.23 \mathrm{GeV}$. The same data sample would also provide better determinations of the SM $\tau$-lepton parameters, stringent tests of lepton-flavor universality in weak interactions, and shed light on the longstanding anomaly in the measurement of $g-2$ of muon. For instance, for the decay rate of the lepton flavor violation process $\tau^{-} \rightarrow \mu^{-} \gamma$, the current upper limits are a few times $10^{-8}$. At STCF one expects to achieve a sensitivity of a few times $10^{-9}$ with $10 \mathrm{ab}^{-1}$ data at $4.23 \mathrm{GeV}$.

\section{Hyperon decays}

The existence of CP violation in strange, charm and bottom meson decays is well established [18]. However, no CP-violating effect has been observed in the baryon sector so far. Promising channels for searching for new sources of $\mathrm{CP}$ violating are weak decays of the $\Lambda$ and $\Xi$ hyperons, where CP-violation effects in the SM are small but effects of new, beyond-the-SM interactions could be large. Elegant measurements can be performed with high statistics samples of quantum-entangled hyperon-antihyperon pair events [3] that are produced via $J / \psi \rightarrow \Lambda \bar{\Lambda}$ and $\Xi \bar{\Xi}$ decays. A one-year STCF run at the $J / \psi$ resonance would produce data samples of 160 (60) million fully reconstructed $J / \psi \rightarrow \Lambda \bar{\Lambda}(\Xi \bar{\Xi})$ events and allow for more than an order of magnitude improvement of the BESIII CP sensitivity. With $\sim 80 \%$ electron beam polarization, this sensitivity would be improved by an additional factor of four.

\section{QCD physics}

The formation of observed hadrons from QCD partons is still not fully understood. Experimentally, an $e^{+} e^{-}$collider is an ideal laboratory to study the hadronization because of the well understood initial state. At STCF, such a study can be performed by measuring the $R$-value for the totally inclusive cross-section, and by measuring the inclusive production of one or two hadrons. The latter will provide important information about various parton fragmentation functions, such as Collins fragmentation functions [19]. Because STCF will run at energy points up to $7 \mathrm{GeV}$, interesting $Q^{2}$-dependent quantities can be studied with high precisions, whose $Q^{2}$ span, as high as $50 \mathrm{GeV}^{2}$, fit well with the future EIC project in USA and the EicC project [20] in China. The study of the Collins fragmentation function is not only important for understanding hadronization, but also crucial for exploring the inner structure of hadrons in semi-inclusive deep inelastic scattering process, where the Collins fragmentation function at STCF can be taken as inputs to EIC and EicC to extract the parton distribution functions. 
Besides inclusive processes, exclusive processes will be also studied at STCF. There are interesting phenomena near the threshold in $e^{+} e^{-} \rightarrow B \bar{B}$, where $B$ indicates a Baryon. These include time-like nucleon form-factors that could be measured for different $Q^{2}$ values with precisions that match the existing measurements in the space-like region. Furthermore, two-photon physics can be explored with high sensitivity to study the production mechanism and properties of hadrons with different quantum numbers, such as $J^{P C}=0^{-+}, 0^{++}, 1^{++}, 2^{-+}$and $2^{++}$.

\section{Summary}

To summarize, STCF covers a very broad physics program, such as QCD tests, hadron spectroscopy, precisely tests of electroweak interactions of the SM and the hunt for new physics beyond. Many of these measurements at STCF will benefit from the clean reaction environment, strictly constrained kinematics and well-controlled systematic uncertainties, which necessitates a detector with excellent performance, especially in identifying the types of different charged particles, detecting low-momentum charged particles and measuring photons. The unprecedented precisions obtained with high luminosity in the energy region $2 \sim 7 \mathrm{GeV}$ will enable us to have a much more in-depth understanding of the challenges facing the SM and hopefully will provide some clues/solutions to them. It will play a crucial role in leading the high intensity frontier of elementary particle physics worldwide.

\section{References}

[1] M. Ablikim et al. [BESIII Collaboration], Nucl. Instrum. Meth. A 614, 345 (2010).

[2] D. M. Asner, et al. Int. J. Mod. Phys. A 24, S1-794 (2009).

[3] M. Ablikim et al. [BESIII Collaboration], Chin. Phys. C 44, 040001 (2020).

[4] H.-P. Peng, "High Intensity Electron Positron Accelerator (HIEPA)", Novosibirsk, Russia, May $21-25,2018$.

[5] Q. Luo, "Preliminary Conceptual Study of Next Generation Tau Charm Factory accelerator at China", in Proc. 8th International Particle Accelerator Conf. (IPAC'17), Copenhagen, Denmark, May 2017, pp. 3436.

[6] X. D. Shi, X. R. Zhou, X. S. Qin and H. P. Peng, JINST 16, P03029 (2021).

[7] M. Ablikim et al. [BESIII Collaboration], Phys. Rev. Lett. 110, 252001 (2013).

[8] M. Ablikim et al. [BESIII Collaboration], Phys. Rev. Lett. 112, 022001 (2014).

[9] M. Ablikim et al. [BESIII Collaboration], Phys. Rev. Lett. 111, 242001 (2013).

[10] M. Ablikim et al. [BESIII Collaboration], Phys. Rev. Lett. 112, 132001 (2014).

[11] M. Ablikim et al. [BESIII], Phys. Rev. Lett. 126, 102001 (2021).

[12] M. Ablikim et al. [BESIII Collaboration], Phys. Rev. Lett. 118, 092001 (2017). 
[13] M. Ablikim et al. [BESIII Collaboration], Phys. Rev. Lett. 112, 092001 (2014).

[14] H. B. Li and X. R. Lyu, [arXiv:2103.00908 [hep-ex]].

[15] Y. S. Amhis et al. (HFLAV Collaboration), arXiv:1909.12524 [hep-ex].

[16] Y. S. Tsai, Phys. Rev. D 51, 3172 (1995).

[17] J. P. Lees et al. [BaBar Collaboration], Phys. Rev. D 85, 031102 (2012) Erratum: [Phys. Rev. D 85, 099904 (2012)].

[18] M. Saur and F. S. Yu, Sci. Bull. 65, 1428 (2020).

[19] M. Ablikim et al. [BESIII Collaboration], Phys. Rev. Lett. 116, 042001 (2016).

[20] D. P. Anderle, et al. [arXiv:2102.09222 [nucl-ex]]. 